\title{
POLY(SODIUM-10-UNDECYLENATE) AS PSEUDO-STATIONARY PHASE IN THE SEPARATION AND QUANTIFICATION OF POLYAROMATIC HYDROCARBONS BY MICELLAR ELECTROKINETIC CHROMATOGRAPHY
}

\author{
*EDUARDO D. PEREIRA ${ }^{1}$, BERNABÉ L. RIVAS ${ }^{2}$ IVÁN PERIC ${ }^{1}$, PAOLA GALLEGOS ${ }^{1}$ AND \\ VALESKA FIGUEROA ${ }^{1}$
}

\author{
${ }^{1}$ Analytical and Inorganic Chemistry Department, Faculty of Chemistry, University of Concepción, Casilla 160-C, Concepción, Chile \\ ${ }^{2}$ Polymer Department, Faculty of Chemistry, University of Concepción, Casilla 160-C, Concepción, Chile
}

(Received: November 30, 2009 - Accepted: February 1, 2010)

\begin{abstract}
In this work we reported the synthesis of different molar mass fraction of poly(sodium-10-undecylenate) (PSU), and its subsequent utilization as pseudostationary phase in the separation of a mix of 4 polyaromatic hydrocarbons (PAHs); naphthalene (NAPH), fluorene (FLU), phenantrene (PHEN) and pyrene (PYR). Concentration and molar mass fraction of PSU were the main variable studied. The analytical parameters; efficiency $(\mathrm{N})$, selectivity $(\alpha)$, and resolution $\left(\mathrm{R}_{\mathrm{s}}\right)$ were calculated to assess the separation capacity. The best conditions for the separation were achieved using a buffer composed by $12.5 \mathrm{mM}$ of each sodium borate and sodium hydrogen phosphate, yielding $\mathrm{pH} 9.2$ in $\mathrm{ACN} / \mathrm{H}_{2} \mathrm{O}(40 / 60)$ solvent, using PSU of molar mass fraction $30-50 \mathrm{kDa}$ in concentration of $0.25 \mathrm{~g} 100$ $\mathrm{mL}^{-1}$. The applied voltage was $25 \mathrm{kV}$.
\end{abstract}

Finally, Limit of Detection (LOD) and Limit of Quantification (LOQ) for 4 PAHs were calculated from the calibration curves in optimal separation conditions.

\section{INTRODUCTION}

A Micellar electrokinetic chromatography (MEKC) is a mode of capillary electrophoresis (CE) that is capable of separating charged and uncharged compounds. However, the use of single surfactants yields several problems due to the dynamic equilibrium that exists between the surfactant monomer and micelle and the high current generated by some charged micelles. Both of these effects lead to band broadening and separation deterioration in $\mathrm{MEKC}^{1-5}$.

One of the most interesting ways to solve these problems is to use polymeric surfactants. The advantages of the last one are based in the fact that the theoretical Critical Micellar Concentration (CMC) is zero and the equilibrium micelle-surfactant is diminished ${ }^{6-8}$.

Moreover, polymeric surfactants are more stable in presence of organic solvent due to the presence of covalent linkage between the unimers and because of their high molar mass can be conveniently used in MEKC-MS applications without background interference from monomer surfactantes of low molecular weights ${ }^{9-11}$.

MEKC uses the same instrumental setup as CE; however, charged organized media, such as micelles are incorporated that act as the separation medium for uncharged solutes. Charged micelles migrate in the electric field at an electrophoretic velocity that is proportional to their charge-to-size ratio. Uncharged solutes with different micelle-water partition coefficients can then separated. Since the separation mechanism is based on differential partitioning, MEKC is viewed as a chromatographic technique with migrating charged micelles acting as pseudostationary phases ${ }^{12}$.

The uncharged analytes are eluted within an elution window $\left(\mathrm{t}_{\mathrm{mc}} / \mathrm{t}_{\mathrm{eo}}\right)$, where $t_{\mathrm{mc}}$ is the migration time of the micelle or pseudostationary phase and $t_{\mathrm{eo}}$ is the migration time of the electrosmotic flow.

Basically, three types of polymeric phases have been used in MEKC. a) polymerized micelles, b) dendrimers, and c) ionic polymers ${ }^{13-15}$.

One of the most succefull polymers used as pseudostationary for separate a wide range of neutral analytes in MEKC is poly(sodium 10-undecylenate) $(\mathrm{PSU})^{16-19}$. However, despite this, there is not systematic studies that relate the molar mass and hydrodynamic radius with the separation capacity of the PUS. Moreover, there is not attempt to quantify neutral analytes simultaneously using this polymer in MEKC.

In the work reported here, we present the synthesis of different molar mass fraction of PSU and its subsequent utilization as pseudostationary phase in the separation of a mix of polyaromatic hydrocarbons (PAHs). The analytical parameters; efficiency $(\mathrm{N})$, selectivity $(\alpha)$, and resolution $\left(\mathrm{R}_{\mathrm{s}}\right)$ were calculated to assess the separation capacity of 4 molar fractions of PSU at different concentrations. The results were correlated with the hydrodynamic radius of PSU in the same separation experimental conditions. Finally, Limit of Detection (LOD) and Limit of Quantification (LOQ) for 4 PAHs were calculated from the calibration curves in optimal separation conditions.

\section{EXPERIMENTAL}

Reagents and chemicals

Undecylenic acid $98 \%$ was obtained from Aldrich and used without further purification. Disodium hydrogen phosphate $\left(\mathrm{Na}_{2} \mathrm{HPO}_{4} 99.995 \%\right)$, disodium tetraborate $\left(\mathrm{Na}_{2} \mathrm{~B}_{4} \mathrm{O}_{7} 99.999 \%\right.$ ), sodium hydroxide $(\mathrm{NaOH}$ pellets $97 \%$ ), naphthalene (NAPH), fluorene (FLU), phenantrene (PHEN) and pyrene (PYR) were purchased from Aldrich, ammonium persulfate $\left(\mathrm{NH}_{4}\right)_{2} \mathrm{~S}_{2} \mathrm{O}_{8} 98 \%$ p.a) was purchased from Merck. HPLC grade acetonitrile (ACN) was obtained from J.T. Baker. All the solutions were prepared using MilliQ quality water.

Apparatus

EKC was conducted using a Model Prince 450 capillary electrophoresis system with UV-Vis Lambda 1010 detector. This CE instrument is equipped with a carousel for automatic injections of the samples and buffers. A fusedsilica capillary, $75 \mathrm{~mm}$ i.d. $x 70 \mathrm{~cm}$ total length, $60 \mathrm{~cm}$ to the detector was used for all runs at $25^{\circ} \mathrm{C}$

The FT-IR spectra are recorded on a Magna Nicolet 550 spectrophotometer.

The thermal stability of the monomer and polymer were studied under nitrogen atmosphere with a Polymer Laboratories Thermal Analyzer STA 625. The heating rate was $10^{\circ} \mathrm{C} / \mathrm{min}$.

The hydrodynamic radius of the different molar mass polymer fraction was measured by a 90Plus Particle Size Analyzer (Brookhaven Corp) equipped with a high power $35 \mathrm{~mW}$ diode laser.

Procedure

Synthesis of Poly(sodium-10-undecylenate) (PSU)

PSU was synthesized by radical polymerization. For that, $10.0200 \mathrm{~g}$ of undecylenic acid was dissolved in a minimum quantity of a $1.0 \mathrm{M} \mathrm{NaOH}$ solution. The sodium undecylenate (SU) solution obtained and $0.5021 \mathrm{~g}$ of ammonium persulfate were placed in a polymerization flask and completed with MilliQ water to $100 \mathrm{~mL}$. The polymerizing mixture was kept at $60^{\circ} \mathrm{C}$ for $48 \mathrm{~h}$. Subsequently the product was purified by dissolving in water and passing through the ultrafiltration membrane with a molecular mass cut-off 3,000 Da. Finally, the product was lyophilized. $6.0607 \mathrm{~g}(57.6 \%$ of yield) of the white powder was obtained. FT-IR spectrum of undecylenic acid shows the absorption bands corresponding to $\mathrm{C}=\mathrm{O}$ of the carboxylic acid group (1707.68 $\left.\mathrm{cm}^{-1}\right)$ and the doubled bond $\mathrm{C}=\mathrm{C}\left(1644.82 \mathrm{~cm}^{-1}\right)$, instead PSU spectrum shows a characteristic absorption band corresponding to $\mathrm{C}=\mathrm{O}$ of carboxylate group (1561.79 $\left.\mathrm{cm}^{-1}\right)$ while the band corresponding to $\mathrm{C}=\mathrm{C}$ disappeared. Thermal stability of $\mathrm{PSU}\left(350^{\circ} \mathrm{C}\right)$ is considerably higher than the undecylenic acid $\left(150^{\circ} \mathrm{C}\right)$.

Membranes and Fractionation

We used polysulfone Amicon membranes with exclusion limits or molar mass cut-off (MMCO) values of 10,000 Da, 30,000 Da, 50,000 Da and 100,000 Da. PSU was dissolved in water and separated into fractions by ultrafiltration through membranes. Finally, the different fractions were lyophilized. Yield of different molar mass fractions of the polymer is shown in table 1. 
Table 1. Yield of different molar mass fractions of poly (sodium-10undecylenate) (PSU)

\begin{tabular}{|c|c|c|}
\hline PSU molar mass fraction/Da & Mass $/ g$ & Percentage/\% \\
\hline $10,000-30,000$ & 0.6970 & 11.5 \\
\hline $30,000-50,000$ & 1.1311 & 18.7 \\
\hline $50,000-100,000$ & 1.7297 & 28.5 \\
\hline$>100,000$ & 2.5029 & 41.3 \\
\hline
\end{tabular}

Separation Capacity of PSU. MEKC experiments.

PSU was tested as pseudo-stationary phase in Capillary Electrophoresis (CE), using a group of test molecules. Its corresponds to a mix of 4 polycyclic aromatic hydrocarbons (PAHs), naphthalene (NAPH), fluorene (FLU), phenantrene (PHEN) and pyrene (PYR). Fraction of molar mass of the polymer, concentration of the polymer, and percentage of acetonitrile added to the solvent were used as variables in this study.

For all MEKC experiments, the capillaries were prepared by flushing with $0.1 \mathrm{M}$ of $\mathrm{NaOH}$ for $1 \mathrm{~h}$, followed by 15 min rinse with milliQ water and finally 5 min of running buffer containing a fraction of PSU polymer. Between injections, the capillary was flushed for 5 min with $0.1 \mathrm{M} \mathrm{NaOH}$, and water for $20 \mathrm{~min}$ followed by running buffer for $3 \mathrm{~min}$. The buffer composition was a mix of $\mathrm{Na}_{2} \mathrm{HPO}_{4} / \mathrm{Na}_{2} \mathrm{~B}_{4} \mathrm{O}_{7} 12.5 \mathrm{mM}$ each, with $\mathrm{pH} 9.2$ prepared in a solvent mix ACN/ $\mathrm{H}_{2} \mathrm{O}$. The sample solution, mix of 4 test molecules $0.4 \mathrm{mM}$ each, was injected hydrodynamically into a capillary over a period of $6 \mathrm{~s}$ with a pressure of 10 mbar. The applied voltage was varied between $20-30 \mathrm{kV}$. The direct detection using a UV-Vis detector adjusted at $254 \mathrm{~nm}$ was performed. The temperature for all runs was kept at $25^{\circ} \mathrm{C}$.

Finally, simultaneous calibration curves were obtained for the 4 PAHs, in the optimal separation conditions. The concentration range studied was $0.5-4.0$ $\mathrm{mM}$

Hydrodynamic radius for different molar mass fraction of PSU

The average of hydrodynamic radius for different molar mass fraction of PSU was measured by dynamic light scattering technique. For this, dilute solution of PSU fraction was prepared in a $\mathrm{Na}_{2} \mathrm{HPO}_{4} / \mathrm{Na}_{2} \mathrm{~B}_{4} \mathrm{O}_{7}$ buffered $\mathrm{ACN} /$ $\mathrm{H}_{2} \mathrm{O}$ solution. The $\mathrm{pH}$ and salt concentration of the buffer solution were adjusted in order to reach the same condition that in the separation capacity experiments. Moreover, the samples were put in a ultrasonic bath and filtered through syringe membrane filter with pore diameter of $0.45 \mu \mathrm{m}$. The time variation of the scattered intensity was measure at $90^{\circ}$, and 5 runs were performed for each sample. All experiments were conducted at $25^{\circ} \mathrm{C}$.

\section{RESULTS AND DISCUSSION}

Poly(sodium-10-undecylenate) (PSU) was synthesized by radical polymerization. Then was purified and fractionated through ultrafiltration membranes. This method produces four molar mass fractions of PSU with high purity (see fig.1 and table 1). The FT-IR spectrum shows the characteristic absorption bands of carboxylate groups and the hydrocarbon chain. Thermal gravimetric analysis (TGA) shows the increase of the thermal stability of PSU compared with the initial monomer.

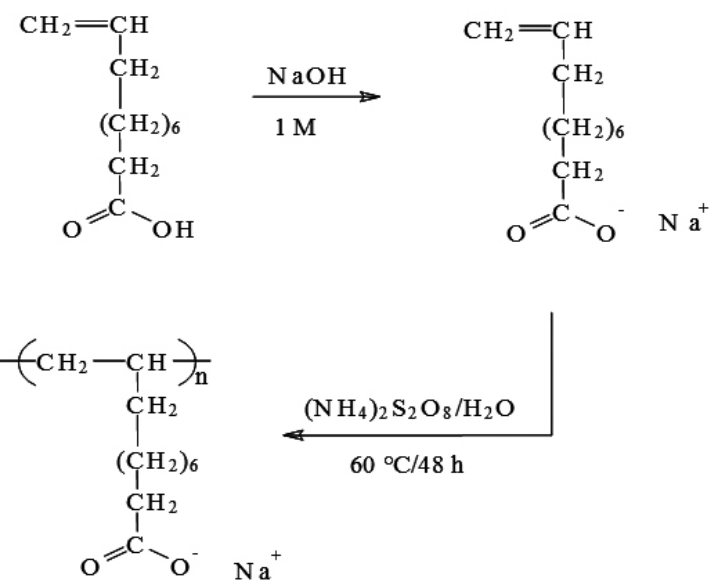

Figure 1. Scheme for the synthesis of poly(sodium 10-undecylenate) (PSU).
PSU have a long hydrocarbon side chain with a polar end group. This leads to propose that the dispersion of this polymer in a polar solvent, like $\mathrm{ACN} / \mathrm{H}_{2} \mathrm{O}$, produce micellar type aggregates with a hydrophobic core. This aggregates will be able to interact differentially with the four PAHs analytes when is added in the running buffer in a CE experiments. These differential interactions, mainly hydrophobic type, between PSU and the 4 PAHs allow its separation.

In order to evaluate the separation capacity of PSU as pseudo-stationary phase, three analytic parameters were calculated; efficiency $(\mathrm{N})$, selectivity (a), and resolution $\left(\mathrm{R}_{\mathrm{s}}\right)$.

Although, these separation parameters are defined for normal Chromatography, is possible to extent the use for MEKC experiments by replacing the retention time $\left(\mathrm{t}_{\mathrm{r}}\right)$ by migration time $\left(\mathrm{t}_{\mathrm{m}}\right)$ in the respective formulas. The effect of the PSU concentration on the separation capacity for the molar mass fraction $30-50 \mathrm{kDa}$ is showed in figure 2.
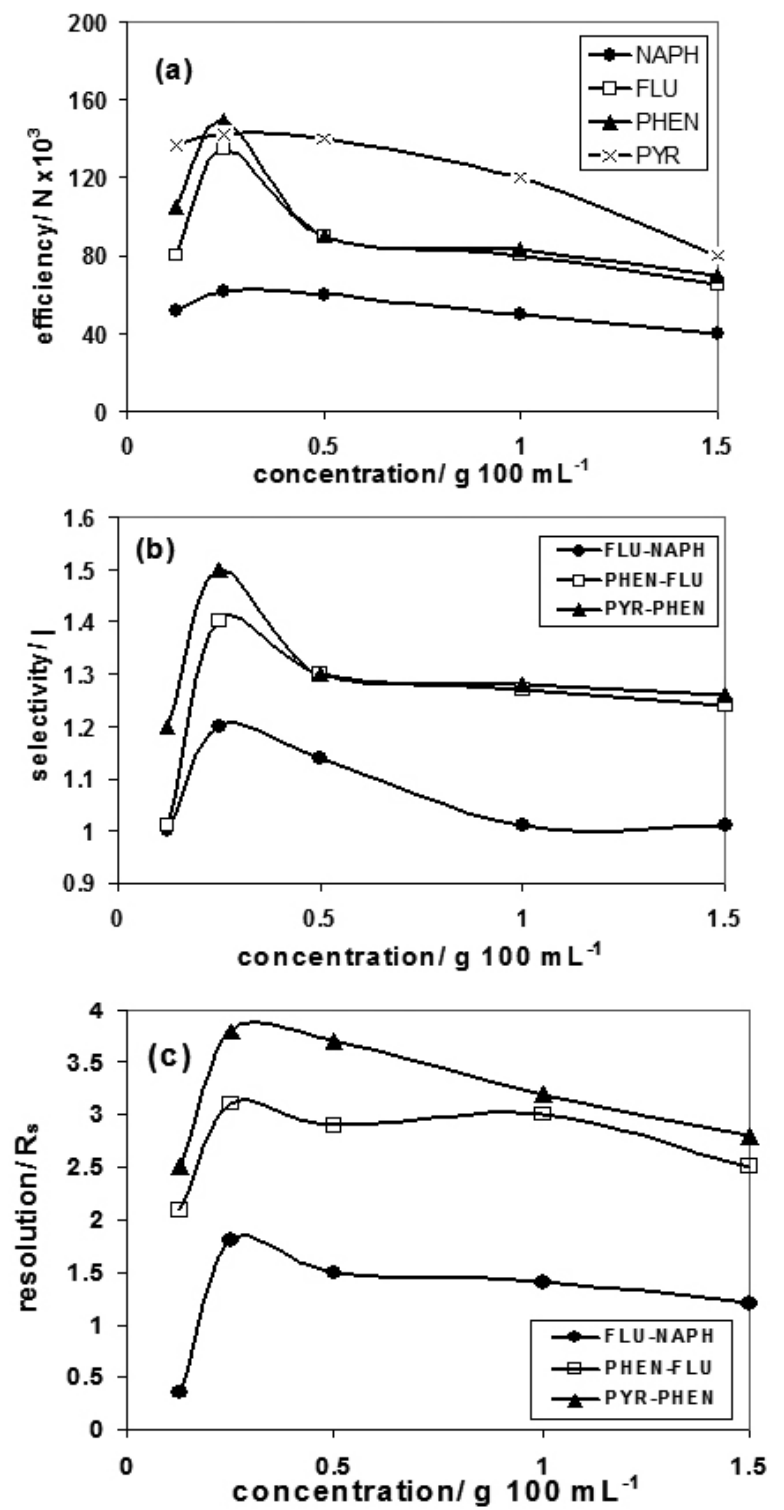

Figure 2. Concentration dependence of PSU in the separation parameters (a) efficiency $(\mathrm{N})$, (b) selectivity $(\alpha)$, and (c) resolution $\left(\mathrm{R}_{\mathrm{s}}\right)$ in the separation of 4 PHAs using PSU as pseudostationary phase in MEKC

In general, the highest values of efficiency were achieved for pyrene in the concentration range studied. However for the 4 PHAs studied, there is a

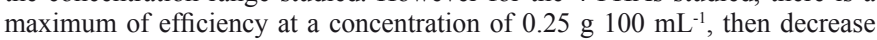
slightly. Consequently, selectivity and resolution calculated for adjacent peaks, also show the same behavior. 
To evaluate the effect of molar mass fraction of PSU in the separation

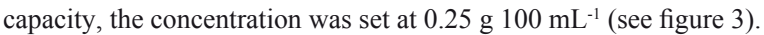
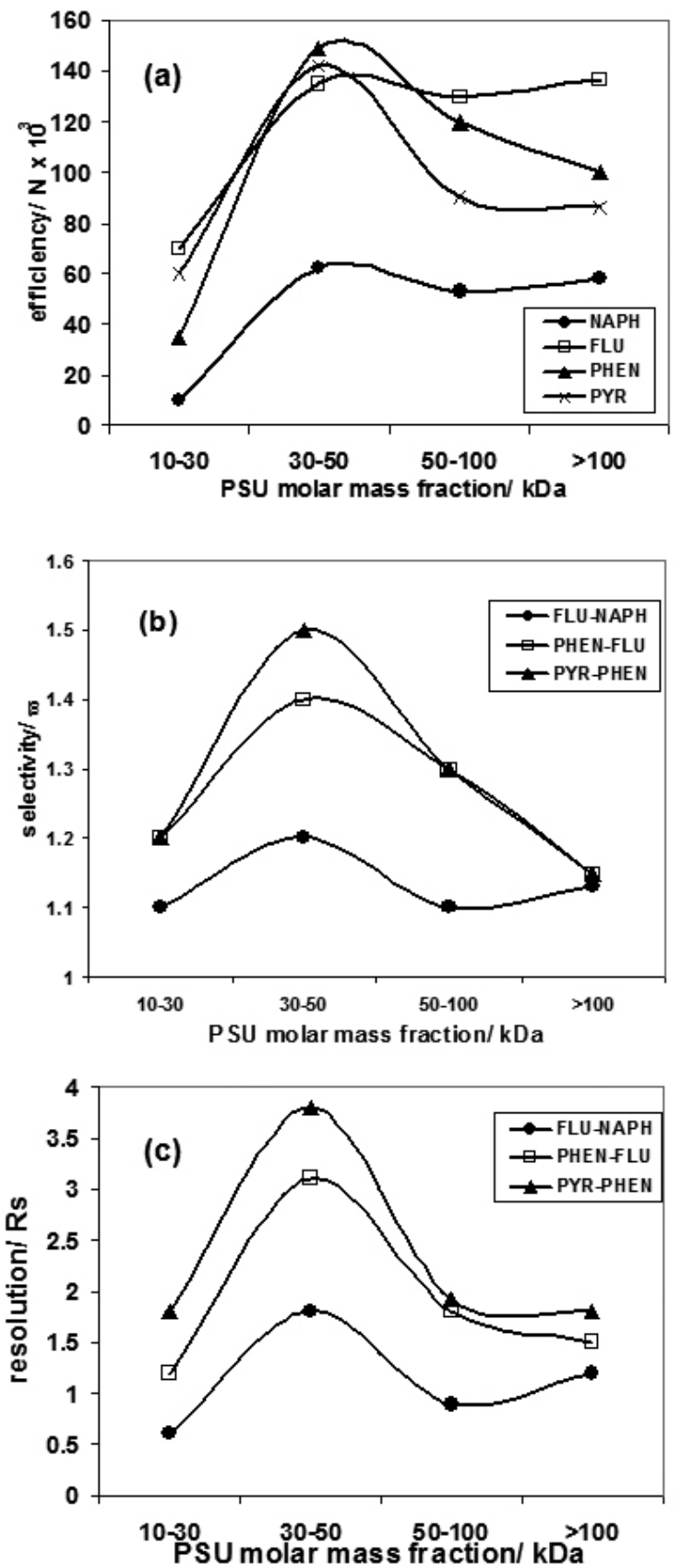

Figure 3. Molar mass fraction of PSU dependence of the separation parameters.

(a) efficiency $(\mathrm{N})$, (b) selectivity $(\alpha)$, and (c) resolution $\left(\mathrm{R}_{\mathrm{s}}\right)$ in the separation of 4 PHAs using PSU as pseudostationary phase in MEKC

The highest values of efficiency were achieved for phenanthrene while the lowest values correspond to naphthalene. Efficiency, selectivity and resolution show a tendency to increase with increasing molar mass fraction of PSU reaching a maximum for the molar mass fraction of 30-50 kDa. To higher values of molar mass fraction, the separation capacity decreases reaching minimum values of $\mathrm{N}, a$, and $\mathrm{R}$, at molar mass fraction $>100 \mathrm{kDa}$.

Considering all variables, the best conditions for the separation were achieved using a buffer composed by $12.5 \mathrm{mM}$ of each sodium borate and sodium hydrogen phosphate, yielding $\mathrm{pH} 9.2$ in $\mathrm{ACN} / \mathrm{H}_{2} \mathrm{O}(40 / 60)$ solvent, using PSU of molar mass fraction $30-50 \mathrm{kDa}$ as pseudostationary phase in

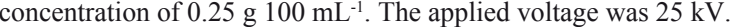
4.

In this condition was obtained the electropherogram showed in the figure

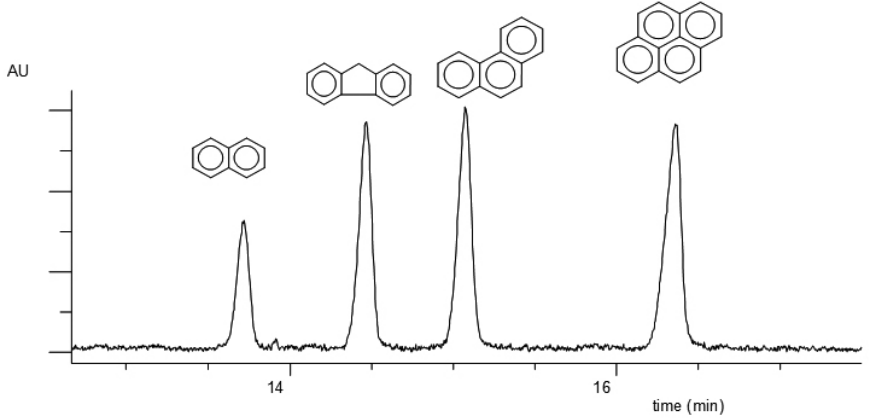

Figure 4. Electropherogram of a mix of 4 PAHs; NAPH, FLU, PHEN, and PYR. Buffer $\mathrm{Na}_{2} \mathrm{HPO}_{4} / \mathrm{Na}_{2} \mathrm{~B}_{4} \mathrm{O}_{7} 12.5 \mathrm{mM} \mathrm{pH}$ 9.2; PSU concentration $0.25 \mathrm{~g}$ $100 \mathrm{~mL}^{-1}$ in $\mathrm{ACN} / \mathrm{H}_{2} \mathrm{O}(40 / 60 \mathrm{v} / \mathrm{v})$; pressure injection 20 mbar for $6 \mathrm{~s}$; applied voltage $+25 \mathrm{kV}$

Here we can see that the separation of the four PAHs; NAPH, FLU, PHEN, and PYR, was achieved in the lower time of analysis, 17 minutes, and with an excellent resolution, selectivity and efficiency.

The efficiency is the ability to produce narrow peaks, meanwhile selectivity and resolution are related with the overlap and separation of the peaks. It means that in the optimal conditions, the pseudostationary phase have higher electrophoretic mobility, low polydispersity and the best differential interactions with the $4 \mathrm{PAHs}$. This must be related with the results obtained from dynamic ligth scattering measurements.

The figure 5 shows the dependence of the hydrodynamic radius of PSU with the molar mass fraction in the same optimal conditions that were used in MEKC experiments.

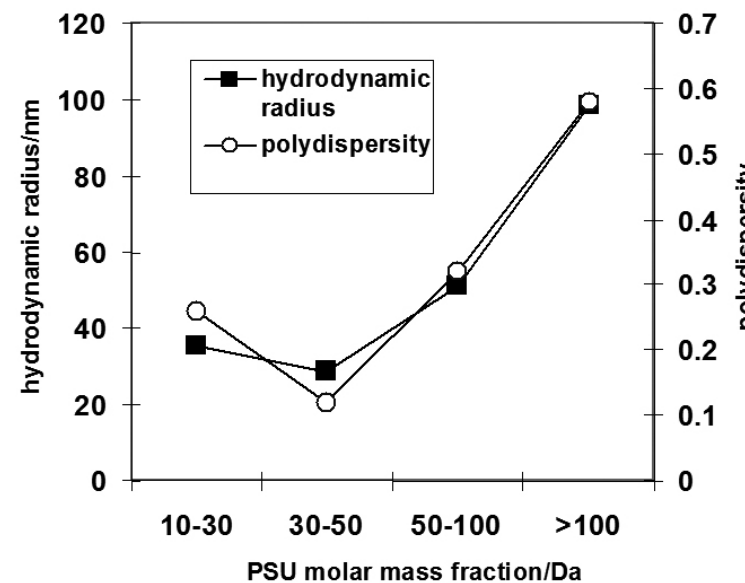

Figure 5. PSU molar mass fraction dependence of the hydrodynamic radius and polydisperisty measured by Dynamic Light Scattering in buffer solution, $\mathrm{Na}_{2} \mathrm{HPO}_{4} / \mathrm{Na}_{2} \mathrm{~B}_{4} \mathrm{O}_{7} 12.5 \mathrm{mM} \mathrm{pH} 9.2$

It shows a minimum value of hydrodynamic radius and polydispersity at molar mass fraction of PSU 30-50 kDa. The hydrodynamic radius is related with the diffusion coefficient through the Stokes-Einstein equation:

$$
D=\frac{k_{B} T}{6 \pi \eta R_{H}}
$$

Where $k_{b}$ is the Boltzman's constant, D is the diffusion coefficient, $\eta$ is the solvent viscosity, $\mathrm{T}$ the absolute temperature, and $\mathrm{R}_{\mathrm{H}}$, the hydrodynamic radius . 
From this equation is easy to see that for lower values of hydrodynamic radius, the diffusion coefficient is higher.

Finally, the concentration dependence with the signal area was studied simultaneously for the 4 PAHs studied in the optimal conditions. For the four calibration curves constructed, the linear correlation coefficient, R, was calculated. In order to check if the obtained values are statistically significant, Student's t-distribution was calculated with $\mathrm{n}=5$ and a confidence interval of $95 \%$. Moreover, based on the statistical error $S_{\gamma / x}$, the standard deviation of the slope $\left(S_{b}\right)$ and coefficient position $\left(S_{a}\right)$ were calculated.

Where no blank has been measured, we can use the calibration data and regression statistics for calculate de LOD and LOQ. In this case, we would use the $y$-intercept (a) and standard deviation of the regression $\left(S_{y / x}\right)$ for to get $Y_{L O D}$ as we can see in eq. 2 :

$$
\mathrm{Y}_{\mathrm{LOD}}=\mathrm{a}+3 S_{\mathrm{y} / \mathrm{x}}
$$

Again, the actual limit-of-detection is the concentration of analyte giving rise to this value. We can therefore obtain the confidence interval for the limitof-detection in the same way as for any interpolated value as shown above. According to that, the Limit of Quantification (LOQ) were calculated for each analyte using the equation 3 .

$$
\mathrm{Y}_{\mathrm{LOQ}}=10 \mathrm{Y}_{\mathrm{LOD}}
$$

Table 2 shows the statistical parameter calculated for each calibration curves. The linear correlation coefficient is acceptable for the 4 analytes and according with the value of $t$ student calculated are statistically significant. The values of LOD and LOQ show that the developed method is highly sensible and are comparable with others techniques like HPLC.

Table 2. Statistical parameters, LOD and LOQ for 4 PAHs obtained from

\begin{tabular}{|c|c|c|c|c|}
\hline $\begin{array}{c}\text { Parameters } \\
\text { obtained from } \\
\text { the Calibration } \\
\text { Curves }\end{array}$ & $\begin{array}{c}\text { Naphthelene } \\
\text { (NAPH) }\end{array}$ & $\begin{array}{l}\text { Fluorene } \\
(F L U)\end{array}$ & $\begin{array}{c}\text { Phenanthrene } \\
\text { (PHEN) }\end{array}$ & $\begin{array}{l}\text { Pyrene } \\
\text { (PYR) }\end{array}$ \\
\hline $\mathrm{R}$ & 0.996 & 0.985 & 0.998 & 0.994 \\
\hline t student & 18.2 & 11.3 & 6.39 & 6.82 \\
\hline$S_{\mathrm{y} / \mathrm{x}}$ & $5.1 \times 10^{-6}$ & $1.8 \times 10^{-5}$ & $1.5 \times 10^{-5}$ & $8 \times 10^{-6}$ \\
\hline$S_{b}$ & $1.8 \times 10^{-6}$ & $1.9 \times 10^{-5}$ & $4.7 \times 10^{-5}$ & $5.9 \times 10^{-6}$ \\
\hline$S_{\mathrm{a}}$ & $3.5 \times 10^{-6}$ & $1.2 \times 10^{-5}$ & $9.9 \times 10^{-6}$ & $5.9 \times 10^{-6}$ \\
\hline LOD & $\begin{array}{c}1.43 \times 10^{-5} \\
\mathrm{mM}\end{array}$ & $\begin{array}{c}4.7 \times 10^{-5} \\
\mathrm{mM}\end{array}$ & $5.0 \times 10^{-5} \mathrm{mM}$ & $2.0 \times 10^{-5}$ \\
\hline LOQ & $\begin{array}{c}1.43 \times 10^{-4} \\
\mathrm{mM}\end{array}$ & $\begin{array}{c}4.7 \times 10^{-4} \\
\mathrm{mM}\end{array}$ & $5.0 \times 10^{-5} \mathrm{mM}$ & $2.0 \times 10^{-4}$ \\
\hline
\end{tabular}
the simultaneous calibration curves.

\section{CONCLUSIONS}

Synthesis of PSU by radical polymerization followed by purification and fractionation in ultrafiltration membranes, allows to obtain 4 fraction of polymeric surfactants for to use them as pseudo-stationary phase in Capillary Electrophoresis (CE) showing a series of advantages.

The optimal conditions of concentration and molar mass fraction of the pseudostationary phases can be explained by the low hydrodinamic radius and low polydispersity measured by Dynamic Ligth Scattering.

Low detection and quantification limits obtained for the studied analytes allow to project these results to quantify real samples.

\section{ACKNOWLEDGEMENTS}

This work was supported by FONDECYT, Grant No 1061018 and

Research Center of Advanced Polymers, CIPA, and CONICYT grant act 130.

\section{REFERENCES}

1. S. Terabe, K. Otsuka, K. Ichikawa, A. Tsuchiya, T. Ando, Anal. Chem., 56, 111, (1984)

2. M.J. Dunn, B.J. Radola, "Advances in Electrophoresis", Horizon Pubs \& Distributors Inc., 1994.

3. J. Vindevogel, P. Sandra, "Introduction to Micellar Electrokinetic Chromatography", Huthig, Heidelberg., 1992.

4. C.A. Monnig, R.T. Kennedy, Anal. Chem., 66, 280R, (1994)

5. R.L St. Claire III, Anal. Chem., 68, 569R, (1996)

6. K. Otsuka, M. Wada, A. Ishisaka, S. Terabe. Anal. Sci., 18, 101, (2002)

7. S.A. Shamsi, C. Akbay, I.M. Warner, Anal. Chem., 70, 3078, (1998)

8. L.H. Judson, I. M. Warner, Rev. Anal. Chem., 18(6), 317, (2000)

9. S.A. Samsi, Anal. Chem., 73, 5103, (2001)

10. C. Akbay, S.A. Rizvi, S.A. Smansi, Anal Chem., 77, 1672, (2005)

11. C. Akbay, N.L. Gill, I.M. Warner, Electrophoresis, 28, 1752, (2007)

12. M.G.Khaledi, "High Performance Capillary Electrophoresis, Theory, Techniques and Applications", John Wiley \& Sons, NewYork, 1998.

13. M. Hamada, A. Dobashi, Anal. Sci., 18, 83, (2002)

14. C.P. Palmer, Electrophoresis, 28, 164, (2007)

15. I. Peric, E. Kenndler, Electrophoresis, 24, 2924, (2003)

16. C.P. Palmer, S. Terabe, Anal. Chem., 69, 1852, (1997)

17. C.P. Palmer, S.J. Terabe, J. Microcol. Sep., 8, 115, (1996)

18. C.P. Palmer, J. Cromatogr. A., 780, 75, (1997)

19. K. Otsuka, M. Wada, A. Ishisaka, S. Terabe, Anal. Sci., 18, 101, (2002) 